\title{
ANALISI DELLA MIGRAZIONE FEMMINILE NIGERIANA IN ITALIA. LA VIOLAZIONE DEI DIRITTI DELLE DONNE VITTIME DI MIGRAZIONE FORZATA E TRATTA A SCOPO DI SFRUTTAMENTO SESSUALE
}

\author{
ANÁLISIS DE LA MIGRACIÓN FEMENINA NIGERIANA EN ITALIA. \\ INFRACCIÓN DE LOS DERECHOS DE LAS MUJERES PARA LAS VICCTIMAS \\ DE MIGRACIÓN FORZADA Y CON EL FIN DE LA EXPLOTACIÓN SEXUAL
}

ANALYSIS OF THE NIGERIAN FEMALE MIGRATION IN ITALY. INFRINGEMENT OF WOMEN'S RIGHTS FOR VICTIMS OF FORCED MIGRATION AND AIMED FOR SEXUAL EXPLOITATION

MICHELA PICCININI*

\begin{abstract}
SOMMARIO
Cosa significa per una donna nigeriana decidere di lasciare il proprio Paese, di migrare, di separarsi dai propri figli, di rivolgersi a dei trafficanti che permettano loro di arrivare in Italia nonostante spesso siano al corrente che saranno coinvolte nel giro della prostituzione? Per capire questo tipo di migrazione forzata e il meccanismo della tratta è importante avere presente la situazione socio-economica e politica della Nigeria, in particolar modo dagli anni '80 in poi quando qui in Italia si è notato per la prima volta il boom di arrivi di immigrate e immigrati nigeriani. È solo comprendendo la situazione che queste donne vivono ogni giorno che si può prendere coscienza di cosa le spinga ad affidarsi a dei trafficanti per compiere questo viaggio, chiamato rotta del Mediterraneo Centrale. È solo immaginando di essere donna in un Paese misogino, povero e corrotto che
\end{abstract}

si può capire perché decidano di affrontare questo esodo durante il quale il loro corpo diventerà un mero pezzo di carne, una materia prima da vendere e comprare. Nessuna di loro però si aspetta quello che realmente accadrà lungo il tragitto e che una volta arrivate a destinazione dovranno attraversare deserti, saranno detenute contro la propria volontà e senza una ragione, saranno picchiate, vendute più volte e stuprate da uomini, trafficanti, ufficiali, miliziani, saranno uccise. La proibizione di schiavitù e tortura sulla loro pelle non verrà applicata; i loro diritti al movimento, alla libertà, al cercare asilo da persecuzioni, alla vita saranno violati ogni giorno.

PAROLE CHIAVE: Migrazione; Sfruttamento; Tratta di esseri umani; Sfruttamento sessuale;Violenza; Misoginia; Violazione dei diritti; Schiavitù

Dottoressa in Psicologia della personalità e delle relazioni interpersonali. Facoltà di psicologia. Università di Padova. Residente in via Rocca 2, Castellarano, Italia.

michela.piccinini@stud.unifi.it 


\section{RESUMEN}

¿Qué significa para una mujer nigeriana decidir abandonar su país, emigrar, separarse de sus hijos, recurrir a traficantes que le permite llegar a Italia a pesar de que a menudo es consciente de que estará involucrada en la prostitución? Para comprender este tipo de migración forzada y el mecanismo de tráfico, es importante tener en cuenta la situación socioeconómica y política en Nigeria, especialmente a partir de la década de 1980 cuando se notó un auge de llegadas de inmigrantes nigerianos aquí en Italia. Es solo al comprender la situación que viven estas mujeres todos los días que uno puede darse cuenta de lo que las impulsa a confiar en los traficantes para hacer este viaje, Ilamado la ruta del Mediterráneo Central. Es solo imaginando que eres una mujer en un país misó gino, pobre y corrupto que puedes entender por qué deciden enfrentar este éxodo durante el cual su cuerpo se convertirá en un simple pedazo de carne, una materia prima para vender y comprar. Ninguna de ellas, sin embargo, espera lo que realmente sucederá en el camino y una vez que lleguen a su destino tendrán que cruzar desiertos, ser retenidas contra su voluntad y sin razón, ser golpeadas, vendidas varias veces y violadas por hombres, traficantes, oficiales, milicianos, serán asesinadas. No se aplicará la prohibición de la esclavitud y la tortura en su piel; sus derechos al movimiento, a la libertad, a buscar asilo de la persecución, a la vida, serán violados todos los días.

PALABRAS CLAVE: migración, explotación, trata de seres humanos, explotación sexual, violencia, misoginia, violación de los derechos, esclavitud.

\section{ABSTRACT}

What does it mean for a Nigerian woman to decide to leave her country, to migrate, to separate from her children, to turn to traffickers who allow them to arrive in Italy even though they are often aware that they will be involved in prostitution? To understand this type of forced migration and the trafficking mechanism, is important to bear in mind the socio-economic and political situation in Nigeria, especially from the 1980s onwards when the arrival boom of Nigerian immigrants and immigrants was noticed in Italy. It is only understanding the situation that these women live every day that one can become aware of what drives them to rely on traffickers to make this journey, called the Central Mediterranean route. It is imagining that you are a woman in a misogynistic, poor and corrupt country that you can understand why they decide to face this exodus during which their body will become a mere piece of meat, a raw material to sell and buy. None of them, however, expects what really will happen along the way and once they arrive at their destination they will have to cross deserts, be held against their will and without a reason, beaten, sold several times and raped by men, traffickers, officers, militiamen, they will be killed. The prohibition of slavery and torture on their skin will not be applied; their rights to the movement, to freedom, to seek asylum from persecution, to life will be violated every day.

KEYWORDS: migration, exploitation, human trafficking, sexual exploitation, violence, misogyny, violation of rights, slavery. 


\section{LA MIGRAZIONE NIGERIANA}

La Nigeria è chiamata lo "Stato africano diasporico" per antonomasia: la storia delle migrazioni nigeriane conta di tre grandi diaspore avvenute durante il secolo scorso. La prima ondata di migrazioni corrisponde a quella del colonialismo britannico durato dal 1914 al 1960 quando la Nigeria diventò indipendente. Questa migrazione prevedeva l'uscita dal Paese dei figli della classe sociale più abbiente che andavano in Europa a finire i loro studi. In Italia ad esempio i primi immigrati con passaporto nigeriano comparvero negli anni '70. Questo tipo di migrazione permise una certa stabilità economica $\mathrm{e}$ diversi matrimoni misti tra nigeriani e italiani. I migranti avevano la possibilità di mantenere i contatti con la famiglia di origine e viaggiare (Cingolani, 2005). La seconda prese luogo dagli anni '60 ai '9o, in concomitanza di gravi perdite economiche nel settore petrolifero, unica fonte di ricchezza del Paese. La Nigeria è formata dalla presenza di diverse etnie, tra le quali, a causa della distribuzione non chiara delle rendite petrolifere, principale fonte di reddito (costituiscono il $98 \%$ delle esportazioni) si sono innescate violente contestazioni. Alla fine degli anni '9o iniziarono gli attentati nel Delta del Niger alle strutture e al personale delle multinazionali petrolifere. Inoltre a partire dalla fine degli anni ' 80 si è assistito ad una ingente migrazione organizzata delle donne nigeriane provenienti da Delta State e Edo State, in particolar modo dalla capitale di Edo, Benin City. Questa migrazione di donne provenienti dagli Stati del Sud era dovuta alla crisi legata al crollo del prezzo del greggio che intaccò le fasce medie della popolazione a partire dalla fine degli anni ' 70 e fu in quegli anni che ebbe inizio ed iniziò ad essere notata la prostituzione nigeriana sulle nostre strade (Cingolani, 2005). La terza, venne causata dai numerosi colpi di Stato che si susseguirono tra gli anni '70 e gli anni '9o. In quegli anni la Nigeria è sempre stata presieduta da governi militari, la forte insicurezza del popolo ha causato fenomeni migratori di qualsiasi classe sociale (Ceccorulli, 2017). Già in quegli anni si poteva notare come consociativismo, insicurezza militare e politica, corruzione e disastro ambientale contribuissero fortemente alla decisione che porta un nigeriano a migrare.

I fattori di spinta (push-factors) e attrazione (pull-factors) che contraddistinguono la migrazione nigeriana attuale sono molteplici e se questi sono sufficienti a far migrare un uomo nigeriano, possiamo solo immaginarci quanto una donna senta il bisogno di emigrare da un Paese misogino dove il tasso di scolarizzazione è pari al $30 \%$, di cui $60 \%$ maschile e $40 \%$ femminile1. Le adolescenti e giovani

1 Convegno Prostituzione e tratta in Italia e in Europa, (2016). Dall'evoluzione del fenomeno alle strategie transnazionali innovative. Atti a cura di Carchedi F. Evoluzione del fenomeno della tratta ai fini di prostituzioni. Il caso della Nigeria, Consortium Parsec di Roma, Roma, 18 Ottobre 2016, https://www.luleonlus.it/wp-content/uploads/2016/11/Dott.-Francesco-CARCHEDI-Area-Ricerche-del-Parsec-Consortium.pdf. 
donne di oggi, ispirate anche dagli esempi occidentali, si oppongono al modello autoritario e patriarcale avuto fino ad ora cercando emancipazione.

Tra i push-factors economici e politici spiccano sicuramente la bassa richiesta di lavoro e l'alta crescita demografica specialmente nelle zone rurali: la maggior parte delle donne vittime di tratta proviene dallo Stato di Edo, in particolare da Benin City, dove la costante e repentina urbanizzazione crea chiari problemi sociali soprattutto in mancanza di strutture e politiche di adattamento; dagli anni '90 ad oggi la popolazione è aumentata del 2000\% (Ceccorulli, 2017). Inoltre l'esodo da queste zone, si innesta a causa della pratica delle espropriazioni forzate da parte delle compagnie petrolifere in accordo con lo Stato, che aumenta ulteriormente la povertà e l'emarginazione sociale, trasformando questa area in una delle più inquinate al Mondo ed è proprio da queste zone che provengono anche le comunità più a rischio di traffici illeciti. Politiche migratorie limitative poi fanno sì che i flussi migratori siano controllati da leggi molto restrittive, di conseguenza le persone pur di poter migrare le scavalcano illegalmente, specialmente le donne. L'ultimo fattore economico è sicuramente la povertà: la Nigeria è uno dei Paesi più poveri al Mondo e le donne sono riconosciute come la categoria sociale più povera.

Altri fattori di spinta culturale e sociale di stampo più contemporaneo intervengo- no poi nella migrazione di queste donne; tra cui il senso alterato delle cure: una volta le famiglie anche estese incoraggiavano la cura e le attenzioni reciproche all'interno della famiglia, ora le persone sono affette dalla "sindrome della ricchezza" e soprattutto zie e zii con a carico le proprie nipoti hanno preso l'abitudine di ricavarne qualcosa, vendendoli e facendo sì che vengano trafficati dalle zone rurali del Paese versi i centri più grossi poi all'estero per ricevere i soldi che $\mathrm{i}$ nipoti venendo sfruttati guadagnano e inviano a "casa". Lacune di informazioni inoltre contribuiscono a rendere il lavoro dei trafficanti più semplice: tante ragazze ancora non sanno cosa accadrà loro una volta giunte in Italia.

Tra i pull-factors emergono lo sfarzo e la ricchezza occidentali esaltate dai media nigeriani come Nollywood ad esempio, intensificano il fenomeno, il che fortifica il presupposto di emancipazione economica delle donne; a quest'ultima contribuisce anche la pressione dei pari che spinge molte donne a mostrare con orgoglio il raggiungimento di un discreto stile di vita e una buona situazione economica. Questo appare evidente da come le figure dei Beento (connazionali arricchitisi in Italia) e delle Italos (maman o ex- prostitute che tornano arricchite dall'Italia) vengano idolatrate dalle case cinematografiche del luogo (come si può notare nella pellicola di Glamour Girls). Come se non bastasse anche la tecnologia e i social networks contribuiscono a questo 
fenomeno, le donne rimaste in Nigeria infatti possono vedere come, sui profili social delle connazionali emigrate, viene esaltato il lusso e il bene-stare: spesso si fanno fotografare vicino a macchine lussuose indossando eleganti vestiti occidentali e questo è il messaggio che arriva nel loro Paese.

Dagli anni '8o la migrazione nigeriana, specialmente delle donne, si può definire con il termine "diretta" o "forzata": diretta si utilizza quando la migrazione non è completamente libera ma nemmeno completamente forzata, ad esempio quando entrano in gioco fattori politici, ambientali, familiari ed economici, ma anche nei casi di traffico di esseri umani (smuggling), ovvero quando un migrante si rivolge a una persona per affrontare un viaggio; il termine forzata si usa quando si fa riferimento al traffico di esseri umani e agli schiavi (trafficking in human beings), agli esiliati, ossia persone che non avevano nessuna intenzione di lasciare il proprio Paese di origine.

Un'altra caratteristica di questa migrazione è l'appartenenza a quella teoria chiamata Network Thoery: un tipo di migrazione che tenderà a espandersi col tempo consolidando i rapporti interpersonali tra connazionali. Questo flusso migratorio veniva e viene tuttora seguito dalle donne perché si tratta di un tipo di migrazione "più sicura", intrapresa da chi ha deciso di raggiungere amici, parenti e/o connazionali in un Paese sconosciuto. La nota negativa di tale migrazione è che col tempo è diventata un trampolino di lancio non solo per i semplici migranti ma anche per la malavita.

\section{LA TRATTA DELLE DONNE NIGERIANE A SCOPO DI SFRUTTAMENTO SESSUALE}

La globalizzazione, ha creato in Italia, così come in tanti altri Paesi, un mercato richiedente una mano d'opera a basso costo in tutta una serie di aree (Ikpeze e Ifmeye, 2015) andando ad incrementare le nuove forme di schiavitù, come lo sfruttamento lavorativo e sessuale. Quest'ultimo è senz'altro rinforzato anche dal forte androcentrismo che intacca la nostra società e dalla grande, se non totale, quantità di potere che è posta in mano agli uomini. Essendo possibile il commercio di ogni singolo prodotto, la globalizzazione, sommata al potere patriarcale della nostra società ha fomentato sempre più la mercificazione del corpo femminile irraggiungibile, sconosciuto, straniero e di conseguenza della prostituzione forzata e di strada, di cui le donne nigeriane rappresentano la fetta più numerosa nel nostro Paese

Secondo un articolo del Journal of Law, Policy and Globalization in Nigeria, si stima un traffico di persone oltre confine che va dalle 600.000 alle 800.000 all'anno, delle quali l'80\% sono donne e di queste un 50\% sono minori d'età. Per quanto riguarda il loro ingresso in Italia, il numero continua ad aumentare esponenzialmen- 
te dal 2011, anno della prima guerra civile libica. Nel 2014 ci sono state 1.454 presenze, per poi aumentare nel 2015 con 5.633 arrivi. Tra il 2015 e il 2016 il numero di sbarchi di donne nigeriane è quasi raddoppiato passando dal 5.633 a 11.0092; l'OIM (2017) stima che almeno l'80\% di queste persone sia vittima di tratta a scopo di sfruttamento sessuale. Il calcolo del numero delle vittime comunque risulta problematico perché non esiste un vero e proprio sistema di raccolta dati, la maggior parte delle informazioni le otteniamo da polizia, uffici immigrazione, guardia di frontiera e dalle varie Organizzazione non governative distribuite sul nostro territorio.

Le donne nigeriane così come le minorenni possono essere vendute dalla famiglia di origine, ingannate da parenti o conoscenti che risiedono già in Italia o decidere loro stesse di intraprendere il viaggio. Indipendentemente dalla volontà o meno queste donne saranno legate ai trafficanti, tramite la sottoscrizione di un accordo, per un periodo $\mathrm{X}$ di tempo, ovvero fino a quando il loro debito "per affrontare il viaggio" non sarà ripagato (inconsapevoli del fatto che questo debito continuerà a crescere). ${ }^{3}$

Una volta preparate alla partenza, i trafficanti, sotto il controllo delle maman (figura

2 Openmigration, Questione di genere: chi e quante sono le donne che chiedono asilo in Italia, https:// openmigration.org/analisi/questione-di-genere-chie-quante-sono-le-donne-che-chiedono-asilo-in-italia/.

3 Nel marzo del 2018 i riti sono stati vietati in Nigeria dalla massima figura religiosa Oba Ewuare II. criminale più vicina alla vittima nello stato di arrivo, ma a volte anche in quello di partenza), fanno svolgere alle vittime un rituale woodoo, chiamato juju, che le assoggetterà psicologicamente e fisicamente. Durante il juju viene richiesto l'aiuto di Mami Wata (Mother of Water), garante dei rapporti contrattuali (EASO, 2015), che viene raffigurata con il volto di una donna dalla pelle chiara, quasi bianca e dai tratti indoeuropei (Beneduce e Taliani, 2001).

Il culto di Mami Wata è legato a pratiche di guarigione e salvifiche ed è considerata come portatrice di bellezza, amante del benessere ma anche di disprezzo per i legami affettivi e che affligge con malattia e morte chiunque trasgredisca le sue proibizioni; nell'esperienza della tratta questo rituale prevede un legame imprescindibile con i propri aguzzini (Santini, 2017).

Il rito juju che viene fatto rivolgendosi a lei solitamente è improntato sull'individualità della vittima e invoca per lei la ricerca della ricchezza e della prosperità. L’idea e il desiderio sempre più presente del benessere europeo fa sì che anche questo rito esiga appunto che i suoi seguaci non contraggano vincoli familiari, pensino al benessere individuale, alla disponibilità di denaro senza controllo da parte della famiglia.

Una volta eseguito il rituale, i corpi di queste donne diventano veri e propri memoriali di questo contratto che sancisce la resti- 
tuzione di un debito insaldabile, di legami impossibili da recidere (Beneduce e Italiani, 2001).

Oltre al rito juju e il reclutamento tradizionale, sembra che negli ultimi anni la criminalità utilizzi anche Internet e le nuove tecnologie per accedere a un largo bacino di vittime potenziali, con il vantaggio di occultare le rispettive attività e di mettere in atto un'ampia serie di reati in un arco di tempo non troppo breve e su scala non troppo ampia. Secondo quanto riferito dagli Stati membri dell'Unione Europea, molte vittime della tratta, in particolare a fini di sfruttamento sessuale o del lavoro, sono reclutate online ${ }^{4}$.

Indipendentemente dalla forma di reclutamento le donne dal momento in cui stringeranno il patto si si ritroveranno in una condizione servile rispetto gli aguzzini e i trafficanti.

Il dominio può avere varie forme, dalla violenza fisica, al ricatto, alla negoziazione perpetuata allo status di sudditanza. Un ruolo specifico è svolto dalla vicinanza dell'aguzzino alla vittima, questa condizione servile infatti sembra sia aumentata quando tra le due parti c'è una relazione di tipo familiare o una co-abitazione in genere. Questo è esattamente ciò che avviene tra prostitute nigeriane e maman (Carchedi, 2003).

4 Commissione Europea, (2016). Relazione 2016 sui progressi compiuti nella lotta alla tratta di esseri umani a norma dell'articolo 20 della direttiva 2011/36/UE concernente la prevenzione e la repressione della tratta di esseri umani e la protezione delle vittime, https://www.penalecontemporaneo. it/upload/1464218179Relazione\%20Tratta\%202016. pdf.
$\mathrm{Su}$ queste donne viene esercitata qualsiasi forma di violenza, partendo dalla psicologica fino ad arrivare a torture fisiche, violenze sessuali e violenza economica.

\section{DIRITTI UMANI VIOLATI}

La prostituzione coatta e forzata, così come il traffico degli esseri umani è considerata una condizione servile e una forma di schiavitù e come tale una violazione dei Diritti umani. È uno stato socio-economico e socio-psicologico che si instaura tra diverse persone, dove le une detengono dominio e potere decisionale sulle altre e ciò ha luogo quando la condotta è attuata mediante violenza, minaccia, inganno, abuso di autorità e/o l'approfittarsi di una situazione di vulnerabilità, inferiorità fisica o psichica o di una situazione di necessità, o mediante la promessa o la dazione di somme di denaro o di altri vantaggi a chi ha autorità sulla persona.

La stragrande maggioranza delle donne che giungono in Italia partendo dalla Nigeria e dalle zone Subsahariane in genere affronta la rotta del Mediterraneo Centrale, attraversando il deserto del Sahara, l'Algeria, la Libia, l’inferno dei migranti, fino alle coste di-Tripoli.

Secondo il Report on the Human Rights situation of Migrants and Refugees in Lybia, quasi la metà delle profughe e delle migranti che passano per la Libia, comprese le bambine, subiscono gravi violazioni, atrocità e abusi spesso più volte 
durante i viaggi, commessi da funzionari pubblici, miliziani e trafficanti, i quali rimangono assolutamente impuniti (OHCHR, 2018).

La Libia non fa parte della Convenzione di Ginevra del 1951 e non ha leggi che differenzino i migranti irregolari dai rifugiati o richiedenti asilo, tutti i migranti quindi ricadono nella categoria di "migranti irregolari”, stando al rapporto del Segretario Generale delle NU del 2019, le donne migranti "sono frequentemente vittime di abusi sessuali ( il $42 \%$ delle donne passate per la Libia dichiara di essere stata stuprata, prostituzione forzata e altre forme di violenza sessuale ad opera di pubblici ufficiali, membri di gruppi armati e trafficanti” (COI, 2019).

Esse continuano ad essere soggette a privazioni della propria libertà (il 49\% dei migranti afferma di essere stato trattenuto contro la propria volontà); detenzione in centri ufficiali e non ufficiali; detenzioni nelle connection houses; tortura; rapimenti e uccisioni.

In Libia non c'è lo stato di diritto e di conseguenza i diritti umani non esistono: le donne diventano pezzi di carne da spartirsi tra miliziani, vengono rese schiave, sfruttate e stuprate, costrette ad abortire innumerevoli volte; la libertà personale viene violata categoricamente. La libertà di movimento, di espressione, il divieto di essere trattenute contro la propria volontà, di essere incarcerate senza giudizio smettono di esistere per queste donne.
La chiusura delle rotte marittime e le conseguenti intercettazioni in mare, in atto dall'anno 2018, hanno causato un aumento delle detenzioni in Libia e diverse persone hanno perso la vita in mare: solo nel mese di Gennaio del 2019 sono morti 200 migranti.

La Convenzione di Istanbul afferma, all'articolo 3 , che la violenza contro le donne è una violazione dei diritti umani ed è mirata a un determinato gruppo sociale che in questo caso è l'appartenenza al genere femminile. Essendo queste donne, vittime di discriminazione e atrocità, durante il loro viaggio e poiché hanno il fondato diritto di sentirsi in pericolo e vittime di persecuzione hanno diritto alla protezione di uno Stato straniero e allo status internazionale di rifugiate dal momento che quello che accade a queste donne è da considerarsi un crimine contro l'umanità e nello specifico contro il genere femminile.

\section{REFERENCIAS BIBLIOGRÁFICAS}

Beneduce, R. e Taliani, S. (2001) Un paradosso ordinato. Possessione, corpi, migrazioni. Antropologia, 1, 15-42.

Carchedi, F. (2003). Il lavoro servile e le nuove schiavitù. FrancoAngeli, Milano.

Ceccorulli, M. (2017). Le nuove migrazioni. Analisi delfenomeno riguardante iflussi che interessano i confini esterni dell'Unione Europea, Dipartimento Relazioni Internazionali, Luglio 2017, Roma, http://www.difesa.it/SMD_/CASD/IM/ CeMiSS/DocumentiVis/Rcerche_da_ 
pubblicare/Ricerche_2017/Ricerca_AI_ SA_02_2016_CECCORULLI_Rid.pdf.

Cingolani, P. (2005). Migranti nigeriani $e$ associazionismo: il caso di Torino.

Commissione Europea (2016). Relazione 2016 sui progressi compiuti nella lotta alla tratta di esseri umani a norma dell'articolo 20 della direttiva 2011/36/UE concernente la prevenzione e la repressione della tratta di esseri umani e la protezione delle vittime, https://www.penalecontemporaneo.it/upload/1464218179Relazione\%20Tratta\%20 2016.pdf.

Convegno Prostituzione e tratta in Italia e in Europa (2016). Dall'evoluzione del fenomeno alle strategie transnazionali innovative. Atti a cura di CARCHEDI F., Evoluzione del fenomeno della tratta ai fini di prostituzioni. Il caso della Nigeria, Consortium Parsec di Roma, Roma, 18 Ottobre 2016, https://www.luleonlus.it/wp-content/uploads/2016/11/ Dott.-Francesco-CARCHEDI-Area-Ricerche-del-Parsec-Consortium.pdf. EASO (2015). Informazioni sui paesi di origine. Nigeria La tratta di donne a fini sessuali. Recuperado de http://www. osservatoriointerventitratta.it/wp-content/uploads/2016/o4/EASO-Nigeria-La-tratta-di-donne-a-fini-sessuali. pdf. https://openmigration.org/analisi/ questione-di-genere-chi-e-quante-sonole-donne-che-chiedono-asilo-in-italia/.

IKPEZE, O. e IFMEYE, S. (2015). Dynamics of Trafficking in Nigerian Women and Globalization. Journal of Law, Policy and Globalization, 39, 34-46.

OHCHR (2018). Desperate and Dangerous: Report on the human rights situation of migrants and refugees in Libya, 20
Diciembre 2018. Recuperado de https:// www.ohchr.org/Documents/Countries/ LY/LibyaMigrationReport.pdf

OIM (2017). La tratta di esseri umani attraverso la rotta del mediterraneo centrale: Dati, Storie e informazioni raccolte dall'Organizzazione mondiale per le migrazioni, Luglio 2017. Recuperado de http://www.osservatoriointerventitratta.it/wp-content/uploads/2017/o7/ RAPPORTO_OIM_Vittime_di_tratta_o.pdf

Openmigration, Questione di genere: chi e quante sono le donne che chiedono asilo in Italia,

Rapporto COI (2019). Libia - detenzione dei migranti, 28 Gennaio 2019. http:// protezioneinternazionale.giur.uniroma3.it/wp-content/uploads/2019/o2/ Rapporto-COI-Libia-detenzione-migranti-28-gennaio-2019.pdf

Santini, A. (2017). Mami Wata: Mother Water, Sirena, Dea, donna e uomo del Voodoo, Mermaids Research Centre. Recuperado de https://mermaidsresearchcenter.wordpress. com/2017/o8/22/mami-wata-motherwater-sirena-dea-donna-e-uomo-delvoodoo/.

\section{PARA CITAR ESTE ARTÍCULO}

Paccinini, M. (2019). Analisi della migrazione femminile nigeriana in Italia. La violazione dei dirriti delle donne vittime de migrazione forzata e tratta a scopo di sfruttamento sessuale. Collectivus, Revista de Ciencias Sociales, 6(2), 159-168.

DOI: http//dx.doi.org/10.15648/Coll.2.2019.10

Recibido: 05/12/2018 Aprobado: 07/03/2019 